\title{
One-Loop Divergences in Simple Supergravity: Boundary Effects
}

\author{
Giampiero Esposito ${ }^{1,2 *}$ and Alexander Yu. Kamenshchik ${ }^{3 \dagger}$ \\ ${ }^{1}$ Istituto Nazionale di Fisica Nucleare, Sezione di Napoli, \\ Mostra d'Oltremare Padiglione 20, 80125 Napoli, Italy \\ 2 Dipartimento di Scienze Fisiche, \\ Mostra d'Oltremare Padiglione 19, 80125 Napoli, Italy \\ ${ }^{3}$ Nuclear Safety Institute, Russian Academy of Sciences, \\ 52 Bolshaya Tulskaya, Moscow 113191, Russia
}

\begin{abstract}
This paper studies the semiclassical approximation of simple supergravity in Riemannian four-manifolds with boundary, within the framework of $\zeta$ function regularization. The massless nature of gravitinos, jointly with the presence of a boundary and a local description in terms of potentials for spin $\frac{3}{2}$, force the background to be totally flat. First, nonlocal boundary conditions of the spectral type are imposed on spin- $\frac{3}{2}$ potentials, jointly with boundary conditions on metric perturbations which are completely invariant under infinitesimal diffeomorphisms. The axial gauge-averaging functional is used, which is then sufficient to ensure self-adjointness. One thus finds that the contributions of ghost and gauge modes vanish separately. Hence the contributions to the one-loop wave function of the universe reduce to those $\zeta(0)$ values resulting from physical modes only. Another set of mixed boundary conditions, motivated instead by local supersymmetry and first proposed by
\end{abstract}

\footnotetext{
*Electronic address: esposito@napoli.infn.it

$\dagger$ Electronic address: grg@ibrae.msk.su
} 
Luckock, Moss and Poletti, is also analyzed. In this case the contributions of gauge and ghost modes do not cancel each other. Both sets of boundary conditions lead to a nonvanishing $\zeta(0)$ value, and spectral boundary conditions are also studied when two concentric three-sphere boundaries occur. These results seem to point out that simple supergravity is not even one-loop finite in the presence of boundaries.

Typeset using REVTEX 


\section{INTRODUCTION}

Although Einstein's general relativity leads to a theory of quantum gravity which is not perturbatively renormalizable [1], the analysis of the semiclassical approximation remains of crucial importance to test the internal consistency of any theory of quantum gravity. For this purpose, it is necessary to achieve a thorough understanding of the problem of boundary conditions in the theory of quantized fields. Indeed, the path-integral representation of the propagator [2], the general theory of the effective action [3], and the recent attempts to define a quantum state of the universe [4-6], provide three relevant examples where the appropriate formulation of boundary conditions plays a crucial role to obtain a well defined model of some properties of quantum gravity.

For gauge fields and gravitation, one may reduce the theory to its physical degrees of freedom by imposing a gauge condition before quantization $[7,8]$, or one may use the FaddeevPopov formalism for quantum amplitudes [9], or the extended-phase-space Hamiltonian formalism of Batalin, Fradkin and Vilkovisky [10]. Moreover, a powerful nondiagrammatic method to perform the one-loop analysis is the one which relies on $\zeta$-function regularization [11]. This is a naturally occurring technique, since semiclassical amplitudes involve definition and calculation of determinants of elliptic, self-adjoint differential operators.

Once these choices are made, there are still many problems which deserve a careful consideration. They are as follows.

(i) Choice of background four-geometry. This may be flat Euclidean four-space, which is relevant for massless theories [12], or the de Sitter four-sphere [13], which is relevant for inflationary cosmology [14], or more general curved backgrounds. The latter appear interesting for a better understanding of quantum field theory in curved space-time.

(ii) Choice of boundary three-geometry. This may consist of two three-surfaces (e.g. two concentric three-spheres), motivated by quantum field theory [15], or just one threesurface (e.g. one three-sphere), motivated by quantum cosmology [4,5], or more complicated examples of boundary three-geometries. 
(iii) Choice of gauge-averaging functional. For example, one may study Lorentz or Coulomb gauge for Euclidean Maxwell theory [16-18], or de Donder or axial gauge for gravitation [19-22], or the noncovariant gauges proposed in Refs. [16,18,23], which take explicitly into account extrinsic-curvature effects.

(iv) Boundary conditions. These may be Dirichlet, Neumann or Robin for scalar fields [24]; local or spectral for massless spin- $\frac{1}{2}$ fields [25-31]; magnetic or electric for Maxwell theory [16-18,23]; local or spectral for spin- $\frac{3}{2}$ potentials [26-31]; completely invariant under infinitesimal diffeomorphisms on metric perturbations [32], or expressed in terms of (complementary) projectors at the boundary [33], or Robin on spatial perturbations and Dirichlet on normal perturbations [21], or manifestly symmetric [34], for linearized gravity.

Since the detailed calculations in Refs. [16-31] have led to the correct values of the oneloop divergences for spin- $\frac{1}{2}$ fields, Euclidean Maxwell theory and Euclidean quantum gravity for various choices of boundary conditions, including all ghost and gauge modes whenever appropriate, the last open problem in this respect was the evaluation of one-loop divergences in simple supergravity in the presence of boundaries. Our paper is thus devoted to a detailed investigation of such an issue.

Simple supergravity is the simplest supersymmetric gauge theory of gravitation [35]. Its action functional consists of the Einstein-Hilbert action, the massless gravitino action [36]

$$
I_{\psi, \widetilde{\psi}} \equiv \frac{1}{2} \int_{M} \epsilon^{\mu \nu \rho \sigma}\left[\widetilde{\psi}_{\mu}^{A^{\prime}} e_{A A^{\prime} \nu} D_{\rho} \psi_{\sigma}^{A}-\psi_{\mu}^{A} e_{A A^{\prime} \nu} D_{\rho} \widetilde{\psi}_{\sigma}^{A^{\prime}}\right] d^{4} x
$$

jointly with real and complex auxiliary fields (which are necessary to close the supersymmetry algebra) and suitable boundary terms depending on the choices of boundary conditions at the bounding three-surfaces. With our notation, which relies on Refs. [36,37], the gravitino potential is represented by the pair of anticommuting, independent (i.e., not related by any conjugation) spinor-valued one-forms $\psi_{\mu}^{A}$ and $\widetilde{\psi}_{\mu}^{A^{\prime}}$. These are obtained by contraction of spinor fields with the spinor version of the tetrad as [12]

$$
\psi_{A \mu}=\Gamma_{A B}^{C^{\prime}} e_{C^{\prime} \mu}^{B},
$$




$$
\widetilde{\psi}_{A^{\prime} \mu}=\gamma_{A^{\prime} B^{\prime}}^{C} e_{C}^{B^{\prime}}
$$

Further details about spinor formalism can be found in our Appendix A, which relies again on Refs. [12,36,37].

The spinor fields $\Gamma$ and $\gamma$ occurring in Eqs. (1.2) and (1.3) are referred to as RaritaSchwinger potentials $[12,38,39]$. They are subject to the infinitesimal gauge transformations $[12]$

$$
\begin{gathered}
\widehat{\Gamma}_{B C}^{A^{\prime}} \equiv \Gamma_{B C}^{A^{\prime}}+\nabla_{B}^{A^{\prime}} \nu_{C}, \\
\widehat{\gamma}_{B^{\prime} C^{\prime}}^{A} \equiv \gamma_{B^{\prime} C^{\prime}}^{A}+\nabla_{B^{\prime}}^{A} \mu_{C^{\prime}} .
\end{gathered}
$$

For the spinor fields $\nu_{C}$ and $\mu_{C^{\prime}}$ to be freely specifiable inside the background four-manifold, the trace-free part of the Ricci tensor has to vanish, jointly with the scalar curvature. Hence the background is forced to be Ricci-flat $[12,40-42]$.

Further restrictions are obtained on considering a local description of $\Gamma$ and $\gamma$ in terms of a second potential $[12,38,39]$. For example, on expressing locally the Rarita-Schwinger potentials as $[12,38,39]$

$$
\begin{gathered}
\gamma_{A^{\prime} B^{\prime}}^{C}=\nabla_{B B^{\prime}} \rho_{A^{\prime}}^{C B}, \\
\Gamma_{A B}{ }^{C^{\prime}}=\nabla_{B B^{\prime}} \theta_{A}^{C^{\prime} B^{\prime}},
\end{gathered}
$$

one finds that the basic equations obeyed by the second potentials $\rho$ and $\theta$ are invariant under infinitesimal gauge transformations with gauge fields $\omega^{D}$ and $\sigma^{D^{\prime}}$ if and only if [12]

$$
\begin{gathered}
\psi_{A F L D} \omega^{D}=0, \\
\widetilde{\psi}_{A^{\prime} F^{\prime} L^{\prime} D^{\prime}} \sigma^{D^{\prime}}=0 .
\end{gathered}
$$

With a standard notation, $\psi_{A F L D}$ and $\widetilde{\psi}_{A^{\prime} F^{\prime} L^{\prime} D^{\prime}}$ are the anti-self-dual and self-dual parts of the Weyl curvature spinor, respectively. Thus, to ensure unrestricted gauge freedom (except 
at the boundary) for the second potentials $\rho$ and $\theta$, one is forced to work in totally flat Euclidean backgrounds. Such a restriction for gravitinos results from the form of the action (1.1), which involves both $\psi_{\mu}^{A}$ and $\widetilde{\psi}_{\mu}^{A^{\prime}}$, jointly with the local description (1.6) and (1.7) and the form of any admissible set of boundary conditions.

Of course, the operator acting on Rarita-Schwinger potentials is a Dirac operator. Within our framework, which deals with positive-definite four-metrics, the Dirac operator is a firstorder elliptic operator which maps primed spinor fields to unprimed spinor fields, and the other way around $[39,43]$. Thus, the specification of the whole gravitino potential at the boundary would lead to an over-determined problem. One thus has a choice of spectral or local boundary conditions for Rarita-Schwinger potentials, and they are both studied in our paper in the presence of three-sphere boundaries $[25,26]$.

Spectral conditions reflect a choice which leads to a well posed classical boundary-value problem. In other words, the massless Rarita-Schwinger potential subject to gauge conditions and linearized supersymmetry constraints is splitted into a regular part and a singular part. The regular part is an infinite sum of modes multiplying harmonics having positive eigenvalues of the intrinsic three-dimensional Dirac operator of the boundary [26,31]. By contrast, the singular part is an infinite sum of modes multiplying harmonics having negative eigenvalues of the intrinsic three-dimensional Dirac operator of the boundary [26,31]. Such an identification relies therefore on a nonlocal operation, i.e., the separation of the spectrum of a first-order elliptic operator into its positive and negative part $[43,44]$.

When the corresponding semiclassical approximation of quantum theory is studied, only half of the gravitino potential is set to zero at the boundary. Bearing in mind the scheme described above, and with the notation of Ref. [31], one writes the spectral boundary conditions on gravitino perturbations in the form

$$
\begin{aligned}
& {\left[\psi_{i(+)}^{A}\right]_{\partial M}=0,} \\
& {\left[\widetilde{\psi}_{i(+)}^{A^{\prime}}\right]_{\partial M}=0,}
\end{aligned}
$$


where the label $(+)$ denotes the part of the perturbation potential corresponding to the regular part of the underlying classical theory. The issue of boundary conditions on $\psi_{0}^{A}$ and $\widetilde{\psi}_{0}^{A^{\prime}}$ will be discussed in Sec. III. To ensure invariance of the boundary conditions (1.10) and (1.11) under the infinitesimal gauge transformations (1.4) and (1.5), we require that

$$
\begin{gathered}
{\left[\nabla_{B}^{A^{\prime}} \nu_{C(+)}\right]_{\partial M}=0,} \\
{\left[\nabla_{B^{\prime}}^{A} \mu_{C^{\prime}(+)}\right]_{\partial M}=0 .}
\end{gathered}
$$

As far as metric perturbations $h_{\mu \nu}$ are concerned, we are interested in setting to zero at the boundary the spatial perturbations [32], i.e.,

$$
\left[h_{i j}\right]_{\partial M}=0
$$

The six boundary conditions (1.14) are invariant under the infinitesimal gauge transformations $[19,20,22]$

$$
{ }^{(\varphi)} h_{\mu \nu} \equiv h_{\mu \nu}+\nabla_{(\mu} \varphi_{\nu)}
$$

if the whole ghost one-form $\varphi_{\nu}$ is set to zero at the boundary:

$$
\left[\varphi_{\nu}\right]_{\partial M}=0
$$

At this stage, the boundary conditions on the normal components $h_{00}$ and $h_{0 i}$ are invariant under (1.15) if and only if the whole gauge-averaging functional $\Phi_{\mu}$ is set to zero at the boundary:

$$
\left[\Phi_{\mu}(h)\right]_{\partial M}=0
$$

As shown in Ref. [22], a choice of gauge-averaging functional which leads to self-adjoint operators on metric perturbations is then the axial, i.e.,

$$
\Phi_{\mu}(h) \equiv n^{\rho} h_{\mu \rho},
$$


where $n^{\rho}$ is the normal to the boundary. Since also the proof of essential self-adjointness of squared Dirac operators with spectral boundary conditions has been recently put on solid ground [45], we have described so far a scheme where the boundary conditions are completely invariant under infinitesimal gauge transformations, the boundary operators on metric perturbations are local and the differential operators on perturbative modes are selfadjoint. Of course, the boundary conditions (1.14) and (1.17) can be re-expressed in terms of tetrad vectors, and the one-loop results coincide with those obtained from the metric formulation.

Another set of boundary conditions is instead motivated by the work in Ref. [46], where it is shown that the spatial tetrad $e_{i}^{A A_{i}^{\prime}}$ and the projection $\left( \pm \widetilde{\psi}_{i}^{A^{\prime}}-\sqrt{2} e_{A^{A^{\prime}}} \psi_{i}^{A}\right)$ transform into each other under half of the local supersymmetry transformations at the boundary. The resulting boundary conditions in one-loop quantum cosmology about flat Euclidean four-space bounded by a three-sphere with Euclidean normal $e_{n^{A^{\prime}}}$ (see (A10)) take the form $[26]$

$$
\sqrt{2}{ }_{e} n_{A}^{A^{\prime}} \psi_{i}^{A}= \pm \widetilde{\psi}_{i}^{A^{\prime}} \text { at } \partial M
$$

jointly with the six boundary conditions (1.14) and the following four boundary conditions on normal components of metric perturbations $[19,20]$ :

$$
\begin{gathered}
{\left[\frac{\partial h_{00}}{\partial \tau}+\frac{6}{\tau} h_{00}-\frac{\partial}{\partial \tau}\left(g^{i j} h_{i j}\right)\right]_{\partial M}=0} \\
{\left[h_{0 i}\right]_{\partial M}=0}
\end{gathered}
$$

where $g$ is the flat background four-metric, and $\tau$ is the Euclidean-time variable, which plays the role of a radial coordinate. Moreover, the ghost one-form for gravitons is subject to mixed boundary conditions, in that the normal component $\varphi_{0}$ obeys Dirichlet conditions [20]:

$$
\left[\varphi_{0}\right]_{\partial M}=0,
$$


and the tangential components obey Robin conditions [20]:

$$
\left[\frac{\partial \varphi_{i}}{\partial \tau}-\frac{2}{\tau} \varphi_{i}\right]_{\partial M}=0
$$

Section II studies the ghost sector in the axial gauge for simple supergravity, when spectral boundary conditions are imposed on gravitino perturbations. Section III is devoted to the corresponding graviton and gravitino contributions. Section IV evaluates the one-loop divergence with the Luckock-Moss-Poletti (LMP) boundary conditions (1.14) and (1.19)(1.23). Section $\mathrm{V}$ performs the one-loop analysis in the axial gauge when two concentric three-sphere boundaries occur, with the same boundary conditions of Secs. II and III. Concluding remarks are presented in Sec. VI, and relevant details are given in the Appendices.

\section{AXIAL GAUGE: GHOST SECTOR}

In this section we study the ghost sector of the gauge-fixed path integral for simple supergravity in the axial gauge. We make such a choice because, in the Barvinsky framework [32] for boundary conditions outlined in the first part of Sec. I, this is the only gauge

condition which ensures self-adjointness of the differential operators acting on perturbative modes [22]. We begin with the gravitino analysis, since the gravitational sector in the axial gauge is thoroughly examined in Ref. [22].

The spinor fields $\Gamma_{A B}^{C^{\prime}}$ and $\gamma_{A^{\prime} B^{\prime}}^{C^{\prime}}$ occurring in Eqs. (1.2) and (1.3) admit the general decomposition into symmetric and antisymmetric parts

$$
\begin{gathered}
\Gamma^{A C C^{\prime}}=\Gamma^{(A C) C^{\prime}}+\frac{1}{2} \epsilon^{A C} \Gamma_{D}^{D C^{\prime}} \\
\gamma^{A^{\prime} C^{\prime} C}=\gamma^{\left(A^{\prime} C^{\prime}\right) C}+\frac{1}{2} \epsilon^{A^{\prime} C^{\prime}} \gamma_{D^{\prime}}^{D^{\prime} C} .
\end{gathered}
$$

In the axial gauge (cf. Eqs. (3.13) and (3.14)), the following spinor fields are set to zero:

$$
\begin{gathered}
\Phi^{A}(\Gamma) \equiv \Gamma^{A C C^{\prime}}{ }_{e} n_{C C^{\prime}}=0 \\
\widetilde{\Phi}^{A^{\prime}}(\gamma) \equiv \gamma^{A^{\prime} C^{\prime} C}{ }_{e} n_{C C^{\prime}}=0
\end{gathered}
$$


This is the purely two-spinor formulation of the gauge conditions studied in Refs. [26,31]. Since the symmetric part of $\Gamma$ may be further split as the sum of two spinor fields having vanishing contraction with ${ }_{e} n_{C C^{\prime}}[31]$, and a third contribution proportional to [37]

$$
\Gamma_{D F^{\prime}}^{D} e^{F^{\prime}(A}{ }_{e} n^{C) C^{\prime}}
$$

and similarly for the $\gamma$-potential, the gauge conditions (2.3) and (2.4) set to zero the traces $\Gamma_{D}^{D F^{\prime}}$ and $\gamma_{D^{\prime}}^{D^{\prime} F}$ of the Rarita-Schwinger potentials.

In the quantum theory via path integrals, however, one performs a gauge-averaging [16] involving the left-hand sides of (2.3) and (2.4). The resulting ghost operators are obtained by studying the transformation of $\Phi^{A}$ and $\widetilde{\Phi}^{A^{\prime}}$ under the infinitesimal gauge transformations (1.4) and (1.5). Hence one finds the ghost operator

$$
\mathcal{D}_{C}{ }^{A} \equiv{ }_{e} n_{C C^{\prime}} \nabla^{A C^{\prime}}
$$

acting on the spinor field $\nu^{C}$, and the ghost operator

$$
\mathcal{F}_{C^{\prime}}^{A^{\prime}} \equiv{ }_{e} n_{C C^{\prime}} \nabla^{C A^{\prime}}
$$

acting on the spinor field $\mu^{C^{\prime}}$. These are four-dimensional operators but, unlike the fourdimensional Dirac operator, they map elements of a spin-space (either primed or unprimed) to elements of the same spin-space.

The ghost fields are now expanded in harmonics on a family of three-spheres centred on the origin as

$$
\begin{aligned}
\nu^{A} & =\sum_{n=0}^{\infty} \sum_{p, q=1}^{(n+1)(n+2)} \alpha_{n}^{p q}\left[m_{n p}(\tau) \rho^{n q A}+\widetilde{r}_{n p}(\tau) \bar{\sigma}^{n q A}\right], \\
\mu^{A^{\prime}} & =\sum_{n=0}^{\infty} \sum_{p, q=1}^{(n+1)(n+2)} \alpha_{n}^{p q}\left[\widetilde{m}_{n p}(\tau) \bar{\rho}^{n q A^{\prime}}+r_{n p}(\tau) \sigma^{n q A^{\prime}}\right]
\end{aligned}
$$

where the $\alpha_{n}$ are block-diagonal matrices with blocks $\left(\begin{array}{cc}1 & 1 \\ 1 & -1\end{array}\right)$, and the harmonics, defined in Ref. [47], obey the eigenvalue equations described in Appendix A. However, unlike Ref. 
[47], different conventions for numerical factors and the dependence on the radial coordinate have been used, for convenience.

Given the eigenfunction expansions

$$
\begin{aligned}
\nu^{A} & =\sum_{\lambda} \mathcal{C}_{\lambda}^{1} \nu_{(\lambda)}^{A}, \\
\mu^{A^{\prime}} & =\sum_{\tilde{\lambda}} \mathcal{C}_{\tilde{\lambda}}^{2} \mu_{(\tilde{\lambda})}^{A^{\prime}},
\end{aligned}
$$

we now study the eigenvalue equations

$$
\begin{gathered}
\mathcal{D}_{C}^{A} \nu_{(\lambda)}^{C}=\lambda \nu_{(\lambda)}^{A}, \\
\mathcal{F}_{C^{\prime}}^{A^{\prime}} \mu_{(\tilde{\lambda})}^{C^{\prime}}=\tilde{\lambda} \mu_{(\tilde{\lambda})}^{A^{\prime}} .
\end{gathered}
$$

These equations are supplemented by the boundary conditions

$$
\begin{gathered}
{\left[\nabla_{B}^{A^{\prime}} \nu_{C(\lambda)}(+)\right]_{\partial M}=0} \\
{\left[\nabla_{B^{\prime}}^{A} \mu_{C^{\prime}(\tilde{\lambda})}(+)\right]_{\partial M}=0,}
\end{gathered}
$$

which ensure the invariance of the spectral boundary conditions (1.10) and (1.11) under the infinitesimal gauge transformations (1.4) and (1.5).

In our flat Euclidean background bounded by a three-sphere, the left-hand side of Eq. (2.11) reduces to

$$
\mathcal{D}_{C}^{A} \nu_{(\lambda)}^{C}=-\frac{1}{2} \frac{\partial}{\partial \tau} \nu_{(\lambda)}^{A}-{ }_{e} n_{C^{\prime}}{ }^{C} e^{A) C^{\prime} i(4)} \nabla_{i} \nu_{C(\lambda)}
$$

by virtue of Eq. (A12). On using the Eqs. (A11), (A13) and (A14) of Appendix A, this yields

$$
\mathcal{D}_{C}^{A} \nu_{(\lambda)}^{C}=-\frac{1}{2}\left(\frac{\partial}{\partial \tau} \nu_{(\lambda)}^{A}-\frac{3}{2 \tau} \nu_{(\lambda)}^{A}\right)-{ }_{e} n_{C^{\prime}}{ }^{(C} e^{A) C^{\prime} i(3)} \nabla_{i} \nu_{C(\lambda)}
$$

Thus, apart from an unessential proportionality factor of $\frac{1}{2}$ which may be absorbed into the definition of the operators (2.5) and (2.6), one finds the following eigenvalue equations for the ghost modes occurring in the expansion (2.7) (see (A15)-(A18)): 


$$
\begin{gathered}
-\left(\frac{d}{d \tau}+\frac{n}{\tau}\right) m_{n p}=\lambda_{n} m_{n p}, \\
-\left(\frac{d}{d \tau}-\frac{(n+3)}{\tau}\right) \widetilde{r}_{n p}=\lambda_{n} \widetilde{r}_{n p} .
\end{gathered}
$$

Moreover, an entirely analogous treatment of Eq. (2.12) yields the eigenvalue equations for the second set of ghost modes in the form

$$
\begin{gathered}
-\left(\frac{d}{d \tau}-\frac{n}{\tau}\right) \widetilde{m}_{n p}=\widetilde{\lambda}_{n} \widetilde{m}_{n p}, \\
-\left(\frac{d}{d \tau}+\frac{(n+3)}{\tau}\right) r_{n p}=\widetilde{\lambda}_{n} r_{n p} .
\end{gathered}
$$

The solutions of Eqs. (2.17)-(2.20) can be written as

$$
\begin{gathered}
m_{n p}\left(\tau, \lambda_{n}\right)=\tau^{-n} e^{-\lambda_{n} \tau} m_{n p}^{0}, \\
\widetilde{r}_{n p}\left(\tau, \lambda_{n}\right)=\tau^{n+3} e^{-\lambda_{n} \tau} \widetilde{r}_{n p}^{0}, \\
\widetilde{m}_{n p}\left(\tau, \widetilde{\lambda}_{n}\right)=\tau^{n} e^{-\widetilde{\lambda}_{n} \tau} \widetilde{m}_{n p}^{0}, \\
r_{n p}\left(\tau, \widetilde{\lambda}_{n}\right)=\tau^{-(n+3)} e^{-\widetilde{\lambda}_{n} \tau} r_{n p}^{0}
\end{gathered}
$$

Note that, unlike the case of a massless spin- $\frac{1}{2}$ field, regularity at the origin makes it necessary to set to zero everywhere the modes $m_{n p}$ and $r_{n p}$.

Contraction of the boundary conditions (2.13) and (2.14) with the Euclidean normal, jointly with the identity in flat Euclidean four-space

$$
e^{n_{B A^{\prime}}} \nabla^{B A^{\prime}} \nu_{(\lambda)}^{C}=-\frac{\partial}{\partial \tau} \nu_{(\lambda)}^{C}
$$

and with the Eqs. (2.22) and (2.23) for the regular modes, is thus found to lead to the discrete spectra

$$
\lambda_{n}=\frac{(n+3)}{a} \quad \forall n \geq 0
$$




$$
\tilde{\lambda}_{n}=\frac{n}{a} \quad \forall n \geq 0
$$

where $a$ is the three-sphere radius, hereafter set to 1 for convenience, since it does not affect the $\zeta(0)$ value. This implies that a first-order, elliptic and positive-definite operator $\mathcal{A}$ exists with spectrum (2.26), for which a $\zeta$-function can be defined as

$$
\zeta_{\mathcal{A}}(s) \equiv \sum_{n=0}^{\infty}(n+1)(n+2)(n+3)^{-s}
$$

and a first-order, elliptic and nonnegative operator $\mathcal{B}$ exists with spectrum (2.27) and finitedimensional null-space, for which a $\zeta$-function can be defined as

$$
\zeta_{\mathcal{B}}(s) \equiv 2+\sum_{n=1}^{\infty}(n+1)(n+2) n^{-s}
$$

where the dimension of the null-space has been included in the definition of the $\zeta$-function, following Ref. [48] (for the opposite view in the literature, see e.g. Ref. [49]). Note that our definitions (2.28) and (2.29) are particular cases of $\eta$-functions [31,43,48], since our operators are first-order but either positive-definite or at least nonnegative. By using Eqs. (B3)-(B6) one thus finds

$$
\begin{gathered}
\zeta_{\mathcal{A}}(0)=\zeta_{H}(-2,3)-3 \zeta_{H}(-1,3)+2 \zeta_{H}(0,3)=-\frac{3}{4}, \\
\zeta_{\mathcal{B}}(0)=2+\zeta_{R}(-2)+3 \zeta_{R}(-1)+2 \zeta_{R}(0)=\frac{3}{4} .
\end{gathered}
$$

Hence the ghost gravitino contribution to the one-loop divergence in the axial gauge vanishes in our flat background bounded by a three-sphere:

$$
\zeta_{\text {ghost }}(0)=\zeta_{\mathcal{A}}(0)+\zeta_{\mathcal{B}}(0)=0
$$

Note that no further ghost fields occur in our calculation (cf. Sec. IV and Appendix A), since the axial gauge already has the effect to reduce the linearized gravitino potential to its two physical degrees of freedom, corresponding to helicities $3 / 2$ and $-3 / 2$ (see also Sec. III). 
The ghost analysis for gravitons in the axial gauge (1.18) is performed in detail in Ref. [22], and we only need to say that the ghost operator turns out to be [22]

$$
\mathcal{F}_{\mu}{ }^{\nu}=\delta_{\mu}^{\nu} n^{\rho} \nabla_{\rho}+n^{\nu} \nabla_{\mu}
$$

By virtue of the Dirichlet boundary conditions (1.16), ghost modes for gravitons are thus found to vanish everywhere. Hence the whole ghost sector of simple supergravity yields a vanishing contribution to the one-loop divergence, when the axial gauges (1.18) and (2.3), (2.4) are imposed.

Our investigation is part of the more general quantization program of field theories in noncovariant gauges [50]. For a thorough treatment of supergravity in the axial gauge in the absence of boundaries, we refer the reader to Ref. [51], where the gauge-fixing term and the corresponding Faddeev-Popov ghost terms are studied in detail using tetrad notation, $\gamma$-matrices and the auxiliary fields.

\section{GRAVITON AND GRAVITINO MODES}

In the Faddeev-Popov path integral for the semiclassical amplitudes of simple supergravity, one has to add a gauge-averaging term to the original Euclidean action for gravitons and gravitinos, jointly with the corresponding ghost term. In Ref. [22] it has been shown that, in the axial gauge, gravitational perturbations corresponding to the eigenvalue $\lambda$ obey the equations

$$
\begin{aligned}
\lambda \nabla^{\nu} h_{(\lambda) \mu \nu} & =\frac{1}{2 \alpha}\left[\left(K_{\mu}^{\rho} n^{\sigma}+K^{\rho \sigma} n_{\mu}\right) h_{(\lambda) \rho \sigma}+n_{\mu} n^{\sigma} \nabla^{\rho} h_{(\lambda) \rho \sigma}\right] \\
& +\frac{1}{2 \alpha}\left[(\operatorname{Tr} K) n^{\sigma} h_{(\lambda) \mu \sigma}+n^{\sigma} n^{\rho} \nabla_{\rho} h_{(\lambda) \mu \sigma}\right] \\
& \left(-\square+\frac{\lambda}{2}\right) h_{(\lambda) \rho}^{\rho}=\frac{1}{2 \alpha} n^{\mu} n^{\nu} h_{(\lambda) \mu \nu}
\end{aligned}
$$

subject to the boundary conditions according to which the whole set of metric perturbations vanishes at the boundary. A unique, smooth and analytic solution of such an elliptic 
boundary-value problem exists, which picks out transverse-traceless perturbations [22]. The three-sphere boundary plays indeed an important role, since the unperturbed extrinsiccurvature tensor $K$ in Eq. (3.1) turns out to be proportional to the induced three-metric in such a case. Thus, the $\zeta(0)$ contribution from gravitons is the one first obtained in Ref. [52]:

$$
\zeta_{T T}(0)=-\frac{278}{45}
$$

Moreover, in flat Euclidean four-space, the square of the Dirac operator on gravitinos is $-\square \delta^{\mu}{ }_{\nu}$. Thus, denoting by $\psi_{(\lambda)}^{\mu}$ the gravitino eigenfunctions belonging to the eigenvalue $\lambda$, by $n^{\mu}$ the normal to the boundary, and bearing in mind that the axial gauge-averaging term for gravitinos can be written as $\frac{1}{2 \alpha} \widetilde{\psi}_{\nu} n^{\nu} n^{\sigma} \psi_{\sigma}$ [51], one finds an eigenvalue equation for gravitino perturbations in the form

$$
\left(-\square \delta_{\nu}^{\mu}+\frac{1}{\alpha} n^{\mu} n_{\nu}\right) \psi_{(\lambda)}^{\nu}=\lambda \psi_{(\lambda)}^{\mu}
$$

Covariant differentiation of (3.4), and its contraction with flat-space $\gamma$-matrices, yield respectively

$$
\begin{aligned}
\nabla_{\mu}\left(-\square \psi_{(\lambda)}^{\mu}\right)+ & \frac{1}{\alpha}\left[(\operatorname{Tr} K) n_{\sigma} \psi_{(\lambda)}^{\sigma}+n^{\mu} K_{\mu \sigma} \psi_{(\lambda)}^{\sigma}+n^{\mu} n_{\sigma} \nabla_{\mu} \psi_{(\lambda)}^{\sigma}\right]=\lambda \nabla_{\mu} \psi_{(\lambda)}^{\mu} \\
& -\square\left(\gamma_{\mu} \psi_{(\lambda)}^{\mu}\right)+\frac{1}{\alpha} \gamma_{\mu} n^{\mu}\left(n_{\sigma} \psi_{(\lambda)}^{\sigma}\right)=\lambda \gamma_{\mu} \psi_{(\lambda)}^{\mu} .
\end{aligned}
$$

Of course, since the normal to the boundary can always be chosen in the form $n^{\mu}=(1,0,0,0)$, the term $n^{\mu} K_{\mu \sigma}$ vanishes, because $K_{00}=K_{0 i}=0$. Thus, denoting by $\Lambda$ the (positive) eigenvalues of the operator $-\square[22]$, and by $T_{\lambda}$ the trace $\gamma_{\mu} \psi_{(\lambda)}^{\mu}$, the system (3.5), (3.6) reduces in flat space to

$$
\begin{gathered}
-\square \nabla_{\mu} \psi_{(\lambda)}^{\mu}+\frac{1}{\alpha}\left[(\operatorname{Tr} K) n_{\sigma} \psi_{(\lambda)}^{\sigma}+n^{\mu} n_{\sigma} \nabla_{\mu} \psi_{(\lambda)}^{\sigma}\right]=\lambda \nabla_{\mu} \psi_{(\lambda)}^{\mu}, \\
(\Lambda-\lambda) T_{\lambda}+\frac{1}{\alpha} \gamma_{\mu} n^{\mu} n_{\sigma} \psi_{(\lambda)}^{\sigma}=0 .
\end{gathered}
$$


In our flat background, the term on the left-hand side of (3.7) involving covariant derivatives of $\psi_{(\lambda)}^{\sigma}$ reduces to $\frac{\partial}{\partial \tau} \psi_{(\lambda)}^{0}$. If we now express $n_{\sigma} \psi_{(\lambda)}^{\sigma}$ in terms of $T_{\lambda}$ and $\gamma_{\mu} n^{\mu}$ from (3.8), and insert it into the left-hand side of (3.7), we find that this reduces to a linear functional of $\nabla_{\mu} \psi_{(\lambda)}^{\mu}$ and $T_{\lambda}$. Thus, a solution of (3.7), (3.8) exists which picks out transverse-traceless perturbations which obey the gauge condition everywhere, i.e., $\forall \tau \in[0, a]$. Moreover, uniqueness of the elliptic boundary-value problem with spectral boundary conditions implies that this is the only possible solution. More precisely, since we are studying a covariant quantization scheme, in that the operator in Eq. (3.4) is a four-dimensional elliptic operator on both normal and tangential components of Rarita-Schwinger potentials, the full set of spectral boundary conditions consists of (1.10), (1.11), jointly with nonlocal boundary conditions of the spectral type on $\psi_{0}^{A}$ and $\widetilde{\psi}_{0}^{A^{\prime}}$, i.e.,

$$
\begin{gathered}
{\left[\psi_{0}^{A}(+)\right]_{\partial M}=0} \\
{\left[\widetilde{\psi}_{0}^{A^{\prime}}(+)\right]_{\partial M}=0 .}
\end{gathered}
$$

Equations (3.9) and (3.10) involve half of the spin- $\frac{1}{2}$ modes, i.e., those multiplying harmonics on the three-sphere having positive eigenvalues of the intrinsic three-dimensional Dirac operator $[26,31,45,47]$. It should be stressed that boundary conditions on normal components of spin- $\frac{3}{2}$ potentials are necessary in any covariant quantization scheme (see also Sec. IV). By contrast, the analysis in Ref. [26], where reduction to the physical degrees of freedom was performed before quantization, did not need (3.9) and (3.10), but used gauge conditions and linearized supersymmetry constraints.

The resulting gravitino modes contribute [26,31]

$$
\zeta_{\frac{3}{2}}(0)=-\frac{289}{360}
$$

By virtue of (2.32), (3.3) and (3.11), the full $\zeta(0)$ value for simple supergravity in the axial gauge is (bearing in mind the anticommuting nature of gravitinos)

$$
\zeta(0)=-\frac{278}{45}+\frac{289}{360}=-\frac{43}{8}
$$


which coincides with the value first obtained in Ref. [53], where a simplified analysis was presented, without appreciating the vanishing contribution of both sets of ghost modes.

No extra contributions occur in Eq. (3.12), since the field equations for auxiliary fields are algebraic rather than differential, and as our calculations concern only one-loop effects, we can impose such field equations at this point and ignore higher-order ghost contributions [54]. Note also that, instead of (2.3) and (2.4), we might have chosen our gauge condition in the form

$$
\begin{gathered}
\Gamma^{(A C) C^{\prime}}{ }_{e} n_{C C^{\prime}}=0, \\
\gamma^{\left(A^{\prime} C^{\prime}\right) C}{ }_{e} n_{C C^{\prime}}=0 .
\end{gathered}
$$

The resulting ghost operators are a bit more involved, and the problem deserves careful consideration to check coincidence of $\zeta(0)$ values.

\section{LUCKOCK-MOSS-POLETTI BOUNDARY CONDITIONS}

In Ref. [20] it was proved in detail that, on imposing the LMP boundary conditions (1.14) and (1.20)-(1.23), the full $\zeta(0)$ value for gravitons, including gauge and ghost modes, is

$$
\zeta(0)_{\text {gravitons }}=-\frac{758}{45}
$$

This result was obtained by using the analytic algorithm of Ref. [13], and it was confirmed by using the recently corrected geometric formulas for the asymptotic heat kernel with mixed boundary conditions $[24,55]$. Note that the result (4.1) differs from (3.1), and hence there is no cancellation of contributions of gauge and ghost modes for gravitons with LMP boundary

conditions, on using the de Donder gauge-averaging functional $\Phi_{\mu}^{d D} \equiv \nabla^{\nu}\left(h_{\mu \nu}-\frac{1}{2} g_{\mu \nu} g^{\rho \sigma} h_{\rho \sigma}\right)$ $[20]$.

We now study the LMP boundary conditions (1.19) for gravitino perturbations. For this purpose, it is useful to describe some basic properties of the geometric theory of heat-kernel asymptotics, following Refs. [20,24,55]. 
The Schwinger-DeWitt coefficient $A_{2}$ for the elliptic operator $-D_{\mu} D^{\mu}+X$, where $D_{\mu}$ is a gauge derivative with curvature $F_{\mu \nu}$, can be written as

$$
16 \pi^{2} A_{2}=\int_{M} b_{2} d V+\int_{\partial M} c_{2} d \Sigma
$$

The volume coefficient $b_{2}$ is well known [56], while surface terms depend upon the choice of boundary conditions. We use mixtures of Dirichlet and Robin boundary conditions,

$$
\begin{gathered}
{\left[P_{-} \phi\right]_{\partial M}=0,} \\
{\left[\left(\psi+n^{\sigma} \nabla_{\sigma}\right) P_{+} \phi\right]_{\partial M}=0,}
\end{gathered}
$$

where $P_{ \pm}$are projection operators $[22,24]$. The results can be expressed in terms of polynomials in the curvature tensor $R_{\mu \nu \alpha \beta}$ of the background four-manifold and in terms of the extrinsic-curvature tensor of the boundary (hereafter $R$ is the trace of the Ricci tensor), i.e.,

$$
\begin{aligned}
q & \equiv \frac{8}{3}(\operatorname{Tr} K)^{3}+\frac{16}{3} \operatorname{Tr} K^{3}-8(\operatorname{Tr} K)\left(\operatorname{Tr} K^{2}\right)+4 R(\operatorname{Tr} K) \\
& -8 R_{\mu \nu}\left((\operatorname{Tr} K) n^{\mu} n^{\nu}+K^{\mu \nu}\right)+8 R_{\mu \nu \alpha \beta} K^{\mu \alpha} n^{\nu} n^{\beta}
\end{aligned}
$$

and

$$
p \equiv \operatorname{Tr} K^{3}-(\operatorname{Tr} K)\left(\operatorname{Tr} K^{2}\right)+\frac{2}{9}(\operatorname{Tr} K)^{3} .
$$

For Dirichlet boundary conditions [24],

$$
c_{2}^{D}=\operatorname{Tr}\left[-\frac{1}{360} q+\frac{2}{35} p-\frac{1}{3}\left(X-\frac{1}{6} R\right)(\operatorname{Tr} K)-\frac{1}{2} n^{\sigma} \nabla_{\sigma}\left(X-\frac{1}{6} R\right)+\frac{1}{15} C_{\mu \nu \alpha \beta} K^{\mu \alpha} n^{\nu} n^{\beta}\right],
$$

while, for Robin boundary conditions [24],

$$
\begin{aligned}
c_{2}^{R} & =\operatorname{Tr}\left[-\frac{1}{360} q+\frac{2}{45} p-\frac{1}{3}\left(X-\frac{1}{6} R\right)(\operatorname{Tr} K)+\frac{1}{2} n^{\sigma} \nabla_{\sigma}\left(X-\frac{1}{6} R\right)-\frac{4}{3}\left(\psi-\frac{1}{3}(\operatorname{Tr} K)\right)^{3}\right. \\
& +2\left(X-\frac{1}{6} R\right) \psi+\left(\psi-\frac{1}{3}(\operatorname{Tr} K)\right)\left(\frac{2}{45}(\operatorname{Tr} K)^{2}-\frac{2}{15}\left(\operatorname{Tr} K^{2}\right)\right) \\
& \left.+\frac{1}{15} C_{\mu \nu \alpha \beta} K^{\mu \alpha} n^{\nu} n^{\beta}\right] .
\end{aligned}
$$


For mixed boundary conditions $[24,55]$,

$$
\begin{aligned}
c_{2} & =\operatorname{Tr}\left[P_{+} c_{2}^{R}+P_{-} c_{2}^{D}-\frac{2}{15}\left(P_{+; i}\right)\left(P_{+}^{; i}\right)(\operatorname{Tr} K)-\frac{4}{15}\left(P_{+; i}\right)\left(P_{+; j}\right) K^{i j}\right. \\
& \left.+\frac{4}{3}\left(P_{+; i}\right)\left(P_{+}^{; i}\right) P_{+} \psi-\frac{2}{3} P_{+}\left(P_{+}^{; i}\right) n^{\mu} F_{i \mu}\right],
\end{aligned}
$$

where Latin indices run from 1 to 3 and Greek indices run from 0 to $3[20,24,55]$.

Following Ref. [57] we now consider the gravitino action in the Euclidean form

$$
S_{3 / 2}=\frac{1}{2} \int_{M} d^{4} x \sqrt{g} \varepsilon^{\alpha \beta \gamma \delta} \tilde{\psi}_{\alpha} \gamma_{5} \gamma_{\beta} \nabla_{\gamma} \psi_{\delta}
$$

where torsion terms are neglected since we are interested in one-loop calculations $[31,37,54]$. To fix the gauge condition we use the change of variables

$$
\psi_{\mu} \equiv \kappa_{\mu}-\frac{1}{2} \gamma_{\mu} \gamma^{\alpha} \kappa_{\alpha}
$$

Hence the gauge-fixed action including ghosts becomes [54]

$$
S_{3 / 2}=\int_{M} d^{4} x \sqrt{g}\left[\frac{1}{2} \tilde{\kappa}_{\mu} \widehat{\nabla} \kappa^{\mu}+\tilde{\epsilon} \widehat{\nabla} \epsilon+\frac{1}{2} \tilde{\eta} \widehat{\nabla} \eta\right]
$$

where the hat denotes contraction with $\gamma$-matrices according to the standard rule $\widehat{\nabla} \equiv \gamma_{\rho} \nabla^{\rho}$. Here, $\epsilon, \tilde{\epsilon}$ are the usual Faddeev-Popov ghost fields while $\eta$ is the Nielsen-Kallosh ghost field [58,59] (see also Appendix A). All these ghost fields behave as bosonic fields, since gravitinos have a Grassmann nature $[35,54,58,59]$. To evaluate the Schwinger-DeWitt coefficient $A_{2}$ for fermionic fields we use the familiar identity $\log \operatorname{det} A=\frac{1}{2} \log \operatorname{det}\left(A^{+} A\right)$ for the $\operatorname{Dirac}$ operator $A$. This makes it possible to use squared fermionic operators, which reduce to the standard Laplace operator in flat Euclidean four-space.

Let us now define the projectors for spin- $1 / 2$ and spin-3/2 fields. Projectors for a spin- $1 / 2$ field have the following form:

$$
P_{ \pm} \equiv \frac{1}{2}\left(1 \pm i \gamma_{5} \hat{n}\right)
$$

while the (mixed) boundary conditions for this field are [57]

$$
\left[P_{-} \phi\right]_{\partial M}=0
$$




$$
\left[\left(\frac{1}{2} \operatorname{Tr} K+n_{\alpha} \nabla^{\alpha}\right) P_{+} \phi\right]_{\partial M}=0
$$

Note that the square of our $\gamma_{5}$ is 1 , to ensure that (4.12) is a projector. Thus, since the square of $\left(\gamma_{0}\right)_{E}$, the Euclidean $\gamma_{0}$, is also 1, one has to take

$$
\gamma_{5}=\left(\gamma_{0}\right)_{E} \gamma_{1} \gamma_{2} \gamma_{3}=i\left(\gamma_{0}\right)_{L} \gamma_{1} \gamma_{2} \gamma_{3}=\left(\gamma_{5}\right)_{L}
$$

i.e., $\gamma_{5}$ coincides with its Lorentzian counterpart.

For a spin-3/2 field we have the following projectors [57]:

$$
\left(\mathcal{P}_{ \pm}\right)_{\nu}^{\mu} \equiv Q_{\nu}^{\mu} P_{\mp}+n^{\mu} n_{\nu} P_{ \pm}
$$

where $Q_{\nu}^{\mu} \equiv \delta_{\nu}^{\mu}-n^{\mu} n_{\nu}$ [22], and $P_{ \pm}$are the projectors defined in (4.12). The LMP boundary conditions for the massless spin-3/2 field are [57]

$$
\begin{gathered}
{\left[\mathcal{P}_{-} \kappa_{\mu}\right]_{\partial M}=0} \\
{\left[\left(\frac{1}{2}(\operatorname{Tr} K) \delta_{\nu}^{\mu}+i \gamma_{5} \widehat{K}^{\mu} n_{\nu}+i \gamma_{5} n^{\mu} \widehat{K}_{\nu}+n_{\alpha} \nabla^{\alpha} \delta_{\nu}^{\mu}\right) \mathcal{P}_{+} \kappa_{\mu}\right]_{\partial M}=0}
\end{gathered}
$$

where $\widehat{K}^{\mu} \equiv K^{\mu \nu} \gamma_{\nu}$. The LMP choices (4.13), (4.15), (4.16), jointly with (1.14), (1.20)(1.23), correspond to boundary conditions which are invariant under the BRST form of the supersymmetry transformations, with the exception of the spatial components $h_{i j}$ and $\psi_{i}^{A}$ [57]. Thus, one should bear in mind that the LMP boundary conditions are not completely gauge-invariant [20,22]. Note also that the spatial component of (4.15) corresponds to (1.19), while (4.16) can be obtained by requiring that (4.15) should be preserved under the action of the Dirac operator on $\kappa_{\mu}[31,57]$.

Now one can easily find that in our flat four-manifold with a three-sphere boundary the value of $q$ in Eq. (4.4) is (hereafter, the three-sphere radius is set to 1 for simplicity, since its effect is cancelled by the integration over the boundary in (4.2))

$$
q=16
$$

while Eq. (4.5) leads to 


$$
p=0
$$

Insertion of Eqs. (4.17) and (4.18) into Eq. (4.6) leads to

$$
c_{2}^{D}=-\frac{2}{45}(\operatorname{Tr} I)
$$

With our notation, $(\operatorname{Tr} I)$ involves traces over both spinor and tensor indices, and is equal to 8 for (massless) gravitinos. Moreover, from Eq. (4.7) one finds for the case of Robin boundary conditions

$$
c_{2}^{R}=\frac{58}{45}(\operatorname{Tr} I)-\frac{4}{3}\left(\operatorname{Tr} \Omega^{3}\right)+4\left(\operatorname{Tr} \Omega^{2}\right)-4(\operatorname{Tr} \Omega)
$$

where we have defined $\Omega_{\nu}^{\mu} \equiv \frac{1}{2}(\operatorname{Tr} K) \delta_{\nu}^{\mu}+i \gamma_{5} \widehat{K}^{\mu} n_{\nu}+i \gamma_{5} n^{\mu} \widehat{K}_{\nu}$. Thus, in the case of gravitinos, $\Omega_{\nu}^{\mu}$ is not proportional to the identity, and one has to calculate the following traces:

$$
\begin{gathered}
\operatorname{Tr}{\Omega^{\mu}}_{\mu}=2(\operatorname{Tr} K)(\operatorname{Tr} 1)=6(\operatorname{Tr} 1) \\
\operatorname{Tr}{\Omega^{\mu}}_{\nu} \Omega^{\nu}{ }_{\mu}=\left[(\operatorname{Tr} K)^{2}+2\left(\operatorname{Tr} K^{2}\right)\right](\operatorname{Tr} 1)=15(\operatorname{Tr} 1), \\
\operatorname{Tr} \Omega_{\nu}^{\mu} \Omega_{\rho}^{\nu} \Omega^{\rho}{ }_{\mu}=\left[\frac{1}{2}(\operatorname{Tr} K)^{3}+3(\operatorname{Tr} K)\left(\operatorname{Tr} K^{2}\right)\right](\operatorname{Tr} 1)=\frac{81}{2}(\operatorname{Tr} 1),
\end{gathered}
$$

where $(\operatorname{Tr} 1)$ denotes instead the trace over spinor indices only, and is equal to $2=\frac{1}{4}(\operatorname{Tr} I)$ in our case. Substituting Eqs. (4.21)-(4.23) into Eq. (4.20) one has

$$
c_{2}^{R}=-\frac{289}{90}(\operatorname{Tr} I)
$$

To evaluate $c_{2}$, we also need the spatial components of the four-dimensional covariant derivative of the projector $\mathcal{P}_{+}$:

$$
\left(\mathcal{P}_{+}\right)_{\nu ; j}^{\mu}=i \gamma_{5} \hat{k}_{j}\left(n^{\mu} n_{\nu}-\frac{1}{2} \delta_{\nu}^{\mu}\right)+i \gamma_{5} \hat{n}\left(K_{j}^{\mu} n_{\nu}+n^{\mu} K_{\nu j}\right)
$$

After substitution of Eqs. (4.19), (4.24) and (4.25) into Eq. (4.8) one obtains

$$
c_{2}=-\frac{169}{90}(\operatorname{Tr} I)
$$


Bearing in mind that the gravitino has 8 degrees of freedom one thus finds from (4.2) and $(4.26)$

$$
A_{2 \text { gravitino }}=-\frac{169}{90}
$$

We know also that the value of $\zeta(0)$ for a massless spin- $\frac{1}{2}$ field subject to the local boundary conditions $(4.13)$ is $[24-29]$

$$
\zeta_{1 / 2}(0)=\frac{11}{360}
$$

Thus, combining (4.27), (4.28), and bearing in mind that gravitinos are fermionic while their Faddeev-Popov and Nielsen-Kallosh ghosts are bosonic, one finds

$$
\zeta_{3 / 2}(0)=-\frac{169}{90}-\frac{11}{120}=-\frac{709}{360}
$$

which leads to

$$
\zeta(0)_{N=1} \text { SUGRA }=-\frac{758}{45}+\frac{709}{360}=-\frac{119}{8}
$$

by virtue of (4.1). Thus, simple supergravity is not even one-loop finite in the background with boundary motivated by quantum cosmology [16-31]. Moreover, one can check that, with a naive combination of contributions of different fields in extended supergravity theories, one again fails to obtain exact cancellations of one-loop divergences (cf. Ref. [57]). However, our own understanding of antisymmetric tensor fields and curved backgrounds with boundary for higher-N supergravity models remains insufficient, and hence this appears as an open problem for further research (see also Sec. VI).

\section{TWO-BOUNDARY PROBLEM}

So far we have only studied backgrounds motivated by quantum cosmology, i.e., where one three-surface shrinks to zero, so that only one bounding three-sphere occurs [4,5]. In quantum field theory, however, one deals with a path-integral representation of the propagation amplitude with suitable data on two boundary three-surfaces [15]. Thus, this section 
is devoted to the one-loop analysis of simple supergravity when two concentric three-sphere boundaries occur, with radii $\tau_{-}$and $\tau_{+}$respectively $\left(\tau_{+}>\tau_{-}\right)$. As in Secs. II and III, we use the axial gauge with spectral boundary conditions for gravitinos, and completely gauge-invariant boundary conditions for metric perturbations [20,22,32] .

We know from Ref. [22] and Sec. III that, in the axial gauge, the only surviving graviton modes are transverse-traceless. Hence they contribute [19]

$$
\zeta(0)_{\text {gravitons }}=\zeta_{T T}(0)=-5
$$

while ghost modes for gravitons vanish everywhere [22]. Moreover, the gravitino modes picked out by the axial gauge take the form $[26,31]$

$$
\begin{aligned}
& f_{n}(\tau)=\sqrt{\tau} I_{n+2}(M \tau)+\beta_{n} \sqrt{\tau} K_{n+2}(M \tau), \\
& \tilde{f}_{n}(\tau)=\sqrt{\tau} I_{n+3}(M \tau)-\beta_{n} \sqrt{\tau} K_{n+3}(M \tau),
\end{aligned}
$$

where $\beta_{n}$ are some constants, and the integer $n$ is $\geq 0$. The modes $f_{n}$ should vanish at $\tau=\tau_{+}$, and the modes $\tilde{f}_{n}$ should vanish at $\tau=\tau_{-}$. The full degeneracy with spectral boundary conditions is then $2(n+1)(n+4)$, and the equation obyed by the eigenvalues by virtue of these boundary conditions is

$$
I_{n+2}\left(M \tau_{+}\right) K_{n+3}\left(M \tau_{-}\right)+I_{n+3}\left(M \tau_{-}\right) K_{n+2}\left(M \tau_{+}\right)=0 .
$$

We can now apply the technique described in Ref. [13] and used several times by the authors in recent work [17-21,23,27-29]. Thus, many details can be omitted, and we limit ourselves to say that $\zeta(0)$ for gravitinos has the general structure [13]

$$
\zeta(0)=I_{\log }+I_{\mathrm{pole}}(\infty)-I_{\mathrm{pole}}(0)
$$

where

$$
I_{\log }=0
$$




$$
\begin{aligned}
& I_{\text {pole }}(\infty)=0, \\
& I_{\text {pole }}(0)=0,
\end{aligned}
$$

which implies

$$
\zeta(0)_{\text {gravitinos }}=0
$$

The ghost contribution to $\zeta(0)$ for gravitinos is obtained by setting to zero at the boundaries the first derivatives of the modes (2.21)-(2.24). Bearing in mind that the roles of twiddled and untwiddled modes are interchanged with respect to the spin- $\frac{1}{2}$ analysis of Ref. [47], spectral boundary conditions for ghost modes can be written as follows:

$$
\begin{gathered}
\frac{d m_{n p}}{d \tau}\left(\tau=\tau_{-}\right)=0, \\
\frac{d r_{n p}}{d \tau}\left(\tau=\tau_{-}\right)=0, \\
\frac{d \widetilde{m}_{n p}}{d \tau}\left(\tau=\tau_{+}\right)=0, \\
\frac{d \widetilde{r}_{n p}}{d \tau}\left(\tau=\tau_{+}\right)=0 .
\end{gathered}
$$

Equations (5.9) and (5.12) lead to incompatible solutions for $\lambda_{n}$, i.e., $\lambda_{n}=-\frac{n}{\tau_{-}} \leq 0$ and $\lambda_{n}=\frac{(n+3)}{\tau_{+}}>0$, while Eqs. (5.10) and (5.11) lead to incompatible solutions for $\tilde{\lambda}_{n}$, i.e., $\tilde{\lambda}_{n}=-\frac{(n+3)}{\tau_{-}}<0$ and $\tilde{\lambda}_{n}=\frac{n}{\tau_{+}} \geq 0$. Thus, the boundary conditions (5.9)-(5.12) imply that no non-trivial ghost modes exist, and by virtue of Eqs. (5.1) and (5.8) the full $\zeta(0)$ value in the axial gauge in the two-boundary problem is found to be

$$
\zeta(0)_{N=1} \text { SUGRA }=-5
$$




\section{CONCLUDING REMARKS}

This paper has evaluated one-loop divergences in simple supergravity for two choices of mixed boundary conditions. First, on imposing Barvinsky boundary conditions for gravitons in the axial gauge (1.18), jointly with spectral boundary conditions for gravitinos in the axial gauge (2.3) and (2.4), the full one-loop divergence has been found in Eq. (3.12). This has been achieved by means of a two-spinor analysis of the ghost operators (2.5) and (2.6), jointly with a nontrivial cancellation resulting from the $\zeta$-functions (2.28) and (2.29). This calculation provides a very good example of the role played by finite-dimensional null-spaces in one-loop calculations [48]. The analysis of the ghost sector for gravitons in the axial gauge [22] has also been used.

Second, Luckock-Moss-Poletti (LMP) boundary conditions (1.14), (1.20)-(1.23), (4.13), (4.15) and (4.16) have been studied by means of the geometric formulas for the asymptotic heat kernel with mixed boundary conditions, in the corrected form obtained in Refs. [24,55]. Unlike the boundary conditions studied in Secs. II and III, LMP boundary conditions do not lead to a vanishing contribution of ghost and gauge modes, and the full one-loop divergence is the one given in Eq. (4.30).

Third, the two-boundary problem in the axial gauge for supergravity with spectral boundary conditions has been studied. The resulting one-loop divergence has been obtained in Eq. (5.13).

For pure gravity, the investigation of one-loop divergences in the absence of boundaries was already a formidable task. It was indeed a great achievement of Ref. [60] to calculate all one-loop divergences of pure gravity and of gravitation interacting with scalar particles. In particular, in the former case, it was found in Ref. [60] that no physically relevant divergences remain, since they can all be absorbed in a field renormalization. In quantum supergravity, one might hope that the supersymmetry relating bosonic and fermionic fields improves the finiteness properties [1,31,35,61], and the perturbative analysis becomes more involved. One has then to consider S-matrix elements, topological invariants and boundary 
effects $[1,30,31]$. As far as we know, our paper has presented the first detailed investigation of one-loop effects in the axial gauge with spectral boundary conditions. More generally, the results $(3.12),(4.30)$ and (5.13) seem to point out that simple supergravity is not even one-loop finite in backgrounds with boundary.

Note that, despite the singularity at the origin of our flat background with boundary in Secs. II-IV, the axial gauge leads to well defined calculations since the resulting operators on perturbative modes reduce to the standard covariant operators (e.g. the Laplacian on transverse-traceless tensors). Interestingly, since the axial gauge-averaging functional selects transverse-traceless perturbations, whereas all remaining gauge modes are forced to vanish everywhere, no exact cancellation of all contributions to the one-loop divergence seems to occur in this two-boundary problem. This is quite different from the result found in noncovariant gauges for Euclidean Maxwell theory [17], where the full $\zeta(0)$ value was found to vanish. Thus, more work might be in order to interpret correctly the value obtained in Eq. $(5.13)$.

Moreover, we do not yet know how to evaluate explicitly higher-order effects in perturbation theory in the presence of boundaries (cf. Refs. [61,62]), neither in quantum cosmological backgrounds (where one boundary three-surface shrinks to zero $[4,5]$ ), nor in the familiar field-theoretical case where both boundary three-geometries are actually present. Although higher-order calculations cannot be performed by means of $\zeta$-function techniques, the heat kernel provides, in principle, a nondiagrammatic way to study higher orders. Another relevant problem is given by one-loop calculations in the presence of boundaries for higher- $\mathrm{N}$ supergravity models. These are naturally formulated on backgrounds with a nonvanishing cosmological constant [63-65], and the analytic or geometric features of the one-loop calculation remain unknown.

The form of the graviton and gravitino propagators in the axial gauge with Barvinsky [32] and spectral [26,31] boundary conditions respectively is also unknown, and deserves careful consideration, jointly with the analysis of the full one-loop effective action [66-68].

A further interesting issue might be the re-formulation of our perturbative analysis within 
the framework proposed in Ref. [69], where an effective action for gauge fields and gravitation has been constructed that is gauge-invariant and independent of the choice of gaugeaveraging term, in such a way that new Feynman rules without ghost lines are obtained for the first time.

Thus, many exciting open problems remain, and this seems to add evidence in favor of no end being in sight for cosmology and fundamental physics [31].

\section{ACKNOWLEDGMENTS}

One of us (G.E.) would like to thank Peter D'Eath for suggesting the problem and constant encouragement over nine years. Scientific collaboration with Ivan Avramidi on the axial gauge in Euclidean quantum gravity has been of invaluable help, and correspondence with Klaus Kirsten was also very helpful. The work of A.Y.K. was partially supported by the Russian Foundation for Fundamental Researches through Grant No. 96-02-16220-a, and by the Russian Research Project "Cosmomicrophysics".

\section{APPENDIX A:}

The massless spinor fields occurring in Eqs. (1.2) and (1.3) obey the Rarita-Schwinger equations $[12,38,70,71]$

$$
\begin{gathered}
\epsilon^{B^{\prime} C^{\prime}} \nabla_{A\left(A^{\prime}\right.} \gamma_{\left.B^{\prime}\right) C^{\prime}}^{A}=0, \\
\nabla^{B^{\prime}(B} \gamma_{B^{\prime} C^{\prime}}^{A)}=0, \\
\epsilon^{B C} \nabla_{A^{\prime}(A} \Gamma_{B) C}^{A^{\prime}}=0, \\
\nabla^{B\left(B^{\prime}\right.} \Gamma_{B C}^{\left.A^{\prime}\right)}=0 .
\end{gathered}
$$

Thus, by virtue of Eq. (1.6), the second potential $\rho$ obeys the equation [12] 


$$
\epsilon_{F L} \nabla_{A A^{\prime}} \nabla^{B^{\prime}(F} \rho_{B^{\prime}}{ }^{A) L}+\frac{1}{2} \nabla_{A^{\prime}}^{A} \nabla^{B^{\prime} M} \rho_{B^{\prime}(A M)}+\square_{A M} \rho_{A^{\prime}}{ }^{(A M)}+\frac{3}{8} \square \rho_{A^{\prime}}=0,
$$

where $\square_{A M} \equiv \nabla_{B^{\prime}(A} \nabla_{M)}^{B^{\prime}}$, and $\square \equiv \nabla^{C F^{\prime}} \nabla_{C F^{\prime}}$. Remarkably, if the following first-order equation holds $[12,38,39,72]$ :

$$
\nabla^{B^{\prime}(F} \rho_{B^{\prime}}^{A) L}=0
$$

then (A5) becomes an identity. Thus, the analysis of second potentials is reduced to study Eq. (A6) jointly with [12,39]

$$
\nabla^{B\left(F^{\prime}\right.} \theta_{B}^{\left.A^{\prime}\right) L^{\prime}}=0
$$

Equations (A6) and (A7) are invariant under the infinitesimal gauge transformations

$$
\begin{gathered}
\widehat{\rho}_{B^{\prime}}^{A L} \equiv \rho_{B^{\prime}}^{A L}+\nabla_{B^{\prime}}^{A} \omega^{L}, \\
\widehat{\theta}_{B}^{A^{\prime} L^{\prime}} \equiv \theta_{B}^{A^{\prime} L^{\prime}}+\nabla_{B}^{A^{\prime}} \sigma^{L^{\prime}},
\end{gathered}
$$

if and only if Eqs. (1.8) and (1.9) hold. Thus, to have freely specifiable gauge fields $\omega^{L}$ and $\sigma^{L^{\prime}}$, the Ricci-flat background is further restricted to be totally flat [12].

In our paper, we rely in part on the spinor notation described in detail in Refs. [36,37,47]. Thus, we only briefly describe some basic identities used in performing the ghost analysis in Sec. II. Denoting by $n^{A A^{\prime}}$ the two-spinor version of the Lorentzian normal to the boundary, the corresponding Euclidean normal is defined as

$$
{ }_{e} n^{A A^{\prime}} \equiv-i n^{A A^{\prime}}
$$

The spinor version of the tetrad $e^{a}{ }_{\mu}$ is obtained out of the Infeld-van der Waerden symbols as $e^{A A^{\prime}} \equiv e_{\mu}^{a} \sigma_{a}{ }^{A A^{\prime}}[37]$. In terms of these objects, the following identities are found to hold (cf. Refs. [31,36,37])

$$
\begin{gathered}
e^{n_{A A^{\prime}}{ }_{e} n^{A B^{\prime}}}=-\frac{1}{2} \epsilon_{A^{\prime}}^{B^{\prime}}, \\
{ }_{e} n^{A A^{\prime}} e_{A A^{\prime}}=0
\end{gathered}
$$




$$
\begin{aligned}
& e_{A A^{\prime} i} e^{B B^{\prime} i}=-\epsilon_{A}{ }^{B} \epsilon_{A^{\prime}}^{B^{\prime}}-{ }_{e} n_{A A^{\prime}} e^{n^{B B^{\prime}}}, \\
& { }^{(4)} \nabla_{i} \nu_{C}={ }^{(3)} \nabla_{i} \nu_{C}-\frac{1}{\tau}{ }^{n_{C} D^{\prime}} e_{B}{ }^{D^{\prime}}{ }_{i} \nu^{B},
\end{aligned}
$$

where ${ }^{(3)} \nabla_{i}$ denotes three-dimensional covariant differentiation tangentially with respect to the intrinsic Levi-Civita connection of the boundary. Our $\nabla^{A A^{\prime}}$ operator denotes always four-dimensional spinor covariant derivative: $\nabla^{A A^{\prime}} \equiv e_{\mu}^{A A^{\prime}} \nabla^{\mu}$, where, in particular, $e_{0}^{A A^{\prime}}=$ ${ }_{e} n^{A A^{\prime}}$. Note also that, in our flat background, the Euclidean-time component of covariant differentiation reduces to partial derivative with respect to $\tau$, since the shift vectors vanish, and hence the $\omega_{0}^{A B}$ component of the connection one-forms vanishes [36].

The unbarred harmonics occurring in Eqs. (2.7) and (2.8) obey, $\forall n \geq 0$, the eigenvalue equations

$$
\begin{aligned}
& e^{A A^{\prime} j(3)} \nabla_{j} \rho_{A}^{n q}=-\left(n+\frac{3}{2}\right){ }_{e} n^{A A^{\prime}} \rho_{A}^{n q} \\
& e^{A A^{\prime} j(3)} \nabla_{j} \sigma_{A^{\prime}}^{n q}=-\left(n+\frac{3}{2}\right) e^{A A^{\prime}} \sigma_{A^{\prime}}^{n q} \\
& e^{A A^{\prime} j(3)} \nabla_{j}\left({ }_{e} n^{B}{ }_{A^{\prime}} \rho_{B}^{n q}\right)=\left(n+\frac{3}{2}\right) e n^{A A^{\prime}}\left({ }_{e} n^{B}{ }_{A^{\prime}} \rho_{B}^{n q}\right) \\
& e^{A A^{\prime} j{ }^{(3)}} \nabla_{j}\left({ }_{e} n_{A}^{B^{\prime}} \sigma_{B^{\prime}}^{n q}\right)=\left(n+\frac{3}{2}\right) e^{A A^{\prime}}\left({ }_{e} n_{A}^{B^{\prime}} \sigma_{B^{\prime}}^{n q}\right)
\end{aligned}
$$

The corresponding eigenvalue equations for the barred harmonics $\bar{\rho}_{A^{\prime}}^{n q}$ and $\bar{\sigma}_{A}^{n q}$ can be obtained by complex conjugation of the spinors occurring in (A15)-(A18), when regarded as $S L(2, C)$ spinors. Thus, a sign change occurs on the right-hand side of the barred counterpart of (A15)-(A18) by virtue of the $-i$ factor in (A10) and of reality of the Lorentzian normal, which imply that the Euclidean normal is anti-Hermitian [47].

The readers who are more familiar with $\gamma$-matrix formalism, may find it useful to read the following outline of basic properties. The Rarita-Schwinger potential $\psi_{\mu}$ admits a unique Hodge-like decomposition [54] into its trace part $\frac{1}{4} \gamma_{\mu}\left(\gamma^{\sigma} \psi_{\sigma}\right)$, a longitudinal part $\psi_{\mu}^{L}$ and a transverse-traceless part $\psi_{\mu}^{T T}$, such that 


$$
\psi_{\mu}=\psi_{\mu}^{T T}+\psi_{\mu}^{L}+\frac{1}{4} \gamma_{\mu}\left(\gamma^{\sigma} \psi_{\sigma}\right)
$$

where

$$
\begin{gathered}
\gamma^{\mu} \psi_{\mu}^{L}=0 \\
\psi_{\mu}^{\mathrm{L}=} \nabla_{\mu} \chi-\frac{1}{4} \gamma_{\mu} \gamma^{\sigma} \nabla_{\sigma} \chi \\
\gamma^{\mu} \psi_{\mu}^{T T}=0 \\
\nabla^{\mu} \psi_{\mu}^{T T}=0
\end{gathered}
$$

for some spinor field $\chi$ which is determined up to solutions of the twistor equation $[39,54,65]$. This decomposition is orthogonal in that

$$
\int_{M} g^{\mu \nu}\left(\psi_{\mu}^{L}\right)^{\dagger} \psi_{\nu}^{T T} \sqrt{g} d^{4} x=0
$$

The need for a third ghost in Sec. IV can be understood by pointing out that a massless Rarita-Schwinger field has 8 degrees of freedom, and only 4 of these are removed by the Faddeev-Popov ghosts. Hence one is left with 4 degrees of freedom, instead of the 2 which yields the correct counting of helicity states of a massless particle [58]. More precisely, on imposing the gauge conditions

$$
\begin{aligned}
& \gamma^{\rho} \psi_{\rho}=b, \\
& \widetilde{\psi}_{\lambda} \gamma^{\lambda}=\tilde{b},
\end{aligned}
$$

where $\psi_{\rho}$ and $\widetilde{\psi}_{\rho}$ are independent gauge fields in the Rarita-Schwinger Lagrangian

$$
\mathcal{L}_{c l}^{R S}=-\widetilde{\psi}_{\lambda} \epsilon^{\lambda \mu \nu \rho} \gamma_{\mu} \gamma_{5} D_{\nu} \psi_{\rho}
$$

the path-integral representation of the vacuum to vacuum amplitude turns out to be [58] 


$$
Z=\text { const } \times \int[d \psi][d \widetilde{\psi}](\operatorname{det} \gamma D)^{-2} \delta\left(\gamma^{\rho} \psi_{\rho}-b\right) \delta\left(\widetilde{\psi}_{\lambda} \gamma^{\lambda}-\tilde{b}\right) \mathrm{e}^{-S_{c l}[\psi, \tilde{\psi}]} \int[d g][d \bar{g}]
$$

Finally, this is re-expressed as $[57,58]$

$$
\begin{aligned}
Z & =(\text { const })^{\prime} \times \int[d \psi][d \widetilde{\psi}](\operatorname{det} \gamma D)^{-2}(\operatorname{det} M)^{-1} \\
& \times \exp \left\{-S_{c l}[\psi, \tilde{\psi}]-\frac{1}{2} \int d^{4} x \sqrt{|g|} \widetilde{\psi}_{\rho} \gamma^{\rho} M \gamma^{\lambda} \psi_{\lambda}\right\} .
\end{aligned}
$$

The nontrivial feature is the occurrence of $(\operatorname{det} M)^{-1}$, where, in the background gauge, $M$ takes the form $\gamma^{\lambda} D_{\lambda}$.

At a deeper level, as shown in detail in Ref. [59], Eq. (A29) reflects the need for modified Feynman rules in supergravity. Denoting by $\mathcal{R}_{\alpha}^{i}$ the generators of local gauge transformations in supergravity, they turn out to obey the supercommutation relations $[35,59]$

$$
\left\{\mathcal{R}_{\alpha, j}^{i}, \mathcal{R}_{\beta}^{j}\right\}=\mathcal{R}_{\gamma}^{i} f_{\alpha \beta}^{\gamma}+\eta_{\alpha \beta}^{i j} S_{, j}
$$

where the $f_{\beta \gamma}^{\alpha}$ and $\eta_{\alpha \beta}^{i j}$ are functions of the field variables. One then finds that the ghost action contains quartic ghost couplings (i.e., a term quadratic in the ghost and quadratic in the anti-ghost). The antighost transforms as in the standard BRST case, while the gauge fields and the ghost obey a generalization of the standard BRST transformation [59]. All this leads to modified Ward-Slavnov-Taylor identities which ensure the gauge invariance of the quantum theory.

\section{APPENDIX B:}

In Sec. II, we rely on the Riemann and Hurwitz $\zeta$-functions, defined respectively as [73]

$$
\begin{gathered}
\zeta_{R}(s) \equiv \sum_{n=1}^{\infty} n^{-s}, \\
\zeta_{H}(s, \alpha) \equiv \sum_{m=0}^{\infty}(m+\alpha)^{-s} .
\end{gathered}
$$

These functions have an analytic continuation to the whole complex-s plane as meromorphic functions. A very useful formula yields [73] 


$$
\zeta_{H}(-n, \alpha)=-\frac{B_{n+1}(\alpha)}{(n+1)}
$$

where $B_{n}(x)$ denotes the Bernoulli polynomial of order $n$. In particular, we only need

$$
\begin{gathered}
B_{1}(x)=x-\frac{1}{2}, \\
B_{2}(x)=x^{2}-x+\frac{1}{6}, \\
B_{3}(x)=x^{3}-\frac{3}{2} x^{2}+\frac{1}{2} x .
\end{gathered}
$$

Moreover, we need the following values of the $\zeta$-function defined in $(\mathrm{B} 1): \zeta_{R}(-2)=$ $0, \zeta_{R}(-1)=-\frac{1}{12}, \zeta_{R}(0)=-\frac{1}{2}[73]$. 


\section{REFERENCES}

[1] M.J. Duff, in Supergravity 1981, edited by S. Ferrara and J.G. Taylor (Cambridge University Press, Cambridge, England, 1982).

[2] L.S. Schulman, Techniques and Applications of Path Integration (John Wiley and Sons, New York, 1981).

[3] B.S. DeWitt, in Architecture of Fundamental Interactions at Short Distances, Les Houches, Session XLIV, edited by P. Ramond and R. Stora (North-Holland, Amsterdam, 1987).

[4] J.B. Hartle and S.W. Hawking, Phys. Rev. D 28, 2960 (1983).

[5] S.W. Hawking, Nucl. Phys. B239, 257 (1984).

[6] A. Vilenkin, Phys. Rev. D 33, 3560 (1986).

[7] J. Louko, Phys. Rev. D 38, 478 (1988).

[8] M. Henneaux and C. Teitelboim, Quantization of Gauge Systems (Princeton University Press, Princeton, 1992).

[9] L.D. Faddeev and V. Popov, Phys. Lett. B 25, 29 (1967).

[10] M. Henneaux, Phys. Rep. 126, 1 (1985).

[11] S.W. Hawking, Commun. Math. Phys. 55, 133 (1977).

[12] G. Esposito, G. Gionti, A.Yu. Kamenshchik, I.V. Mishakov, and G. Pollifrone, Int. J. Mod. Phys. D 4, 735 (1995).

[13] A.O. Barvinsky, A.Yu. Kamenshchik, and I.P. Karmazin, Ann. Phys. (N.Y.) 219, 201 (1992).

[14] A. Linde, "Quantum Cosmology and the Structure of Inflationary Universe," invited talk at the joint John Hopkins Workshop (gr-qc/9508019). 
[15] S.W. Hawking, in General Relativity, an Einstein Centenary Survey, edited by S.W. Hawking and W. Israel (Cambridge University Press, Cambridge, England, 1979).

[16] G. Esposito, Class. Quantum Grav. 11, 905 (1994).

[17] G. Esposito and A.Yu. Kamenshchik, Phys. Lett. B 336, 324 (1994).

[18] G. Esposito, A.Yu. Kamenshchik, I.V. Mishakov, and G. Pollifrone, Class. Quantum Grav. 11, 2939 (1994).

[19] G. Esposito, A.Yu. Kamenshchik, I.V. Mishakov, and G. Pollifrone, Phys. Rev. D 50, $6329(1994)$.

[20] G. Esposito, A.Yu. Kamenshchik, I.V. Mishakov, and G. Pollifrone, Phys. Rev. D 52, $3457(1995)$.

[21] G. Esposito and A.Yu. Kamenshchik, Class. Quantum Grav. 12, 2715 (1995).

[22] I.G. Avramidi, G. Esposito, and A.Yu. Kamenshchik, "Boundary Operators in Euclidean Quantum Gravity," (hep-th/9603021).

[23] G. Esposito, A.Yu. Kamenshchik, I.V. Mishakov, and G. Pollifrone, Phys. Rev. D 52, $2183(1995)$.

[24] I.G. Moss and S. Poletti, Phys. Lett. B 333, 326 (1994).

[25] P.D. D'Eath and G. Esposito, Phys. Rev. D 43, 3234 (1991).

[26] P.D. D'Eath and G. Esposito, Phys. Rev. D 44, 1713 (1991).

[27] A.O. Barvinsky, A.Yu. Kamenshchik, I.P. Karmazin, and I.V. Mishakov, Class. Quantum Grav. 9, L27 (1992).

[28] A.Yu. Kamenshchik and I.V. Mishakov, Int. J. Mod. Phys. A 7, 3713 (1992).

[29] A.Yu. Kamenshchik and I.V. Mishakov, Phys. Rev. D 47, 1380 (1993). 
[30] G. Esposito, Int. J. Mod. Phys. D 3, 593 (1994).

[31] G. Esposito, Quantum Gravity, Quantum Cosmology and Lorentzian Geometries, Lecture Notes in Physics, Monographs, Vol. m12 (Springer-Verlag, Berlin, 1994).

[32] A.O. Barvinsky, Phys. Lett. B 195, 344 (1987).

[33] H.C. Luckock, J. Math. Phys. 32, 1755 (1991).

[34] V.N. Marachevsky and D.V. Vassilevich, Class. Quantum Grav. 13, 645 (1996).

[35] P. van Nieuwenhuizen, Phys. Rep. 68, 189 (1981).

[36] P.D. D'Eath and D.I. Hughes, Nucl. Phys. B 378, 381 (1992).

[37] P.D. D'Eath, Phys. Rev. D 29, 2199 (1984).

[38] R. Penrose, in Twistor Theory, edited by S. Huggett (Marcel Dekker, New York, 1994).

[39] G. Esposito, Complex General Relativity, Fundamental Theories of Physics, Vol. 69 (Kluwer, Dordrecht, 1995).

[40] S. Deser and B. Zumino, Phys. Lett. B 62, 335 (1976).

[41] D.Z. Freedman, P. van Nieuwenhuizen, and S. Ferrara, Phys. Rev. D 13, 3214 (1976).

[42] D.Z. Freedman and P. van Nieuwenhuizen, Phys. Rev. D 14, 912 (1976).

[43] G. Esposito, "Dirac Operator and Eigenvalues in Riemannian Geometry," SISSA Lectures (gr-qc/9507046).

[44] M.F. Atiyah, V.K. Patodi, and I.M. Singer, Math. Proc. Cambridge Philos. Soc. 77, 43 (1975).

[45] G. Esposito, H.A. Morales-Técotl, and L.O. Pimentel, Class. Quantum Grav. 13, 957 (1996).

[46] H.C. Luckock and I.G. Moss, Class. Quantum Grav. 6, 1993 (1989). 
[47] P.D. D'Eath and J.J. Halliwell, Phys. Rev. D 35, 1100 (1987).

[48] M.F. Atiyah, V.K. Patodi, and I.M. Singer, Math. Proc. Cambridge Philos. Soc. 79, 71 (1976).

[49] T.R. Taylor and G. Veneziano, Nucl. Phys. B 345, 210 (1990).

[50] G. Leibbrandt, Rev. Mod. Phys. 59, 1067 (1987).

[51] T. Matsuki, Phys. Rev. D 21, 899 (1980).

[52] K. Schleich, Phys. Rev. D 32, 1889 (1985).

[53] P.D. D'Eath and G. Esposito, in Proceedings of the 9th Italian Conference on General Relativity and Gravitational Physics, Capri, Italy 1990, edited by R. Cianci, R. de Ritis, M. Francaviglia, G. Marmo, C. Rubano, and P. Scudellaro (World Scientific, Singapore, 1991).

[54] S.M. Christensen, M.J. Duff, G.W. Gibbons, and M. Rocek, Phys. Rev. Lett. 45, 161 (1980).

[55] D.V. Vassilevich, J. Math. Phys. 36, 3174 (1995).

[56] I.G. Avramidi, Nucl. Phys. B 355, 712 (1991).

[57] S. Poletti, Phys. Lett. B 249, 249 (1990).

[58] N.K. Nielsen, Nucl. Phys. B 140, 499 (1978).

[59] R.E. Kallosh, Nucl. Phys. B 141, 141 (1978).

[60] G. 't Hooft and M. Veltman, Ann. Inst. Henri Poincaré 20, 69 (1974).

[61] P. van Nieuwenhuizen and J.A. Vermaseren, Phys. Lett. B 65, 263 (1976).

[62] S. Deser, J.H. Kay, and K.S. Stelle, Phys. Rev. Lett. 38, 527 (1977).

[63] P.K. Townsend, Phys. Rev. D 15, 2802 (1977). 
[64] P. Breitenlohner and D.Z. Freedman, Ann. Phys. (N.Y.) 144, 249 (1982).

[65] G. Esposito and G. Pollifrone, "Twistors in Conformally Flat Einstein Four-Manifolds," (gr-qc/9507015).

[66] J.S. Dowker, Phys. Lett. B 366, 89 (1996).

[67] K. Kirsten and G. Cognola, Class. Quantum Grav. 13, 633 (1996).

[68] G. Esposito, A.Yu. Kamenshchik, and G. Pollifrone, Class. Quantum Grav. 13, 943 (1996).

[69] B.S. DeWitt and C. Molina-París, "Gauge Theory Without Ghosts," hep-th/9511109).

[70] W. Rarita and J. Schwinger, Phys. Rev. 60, 61 (1941).

[71] P.C. Aichelburg and H.K. Urbantke, Gen. Rel. Grav. 13, 817 (1981).

[72] J. Frauendiener, J. Ghosh, and E.T. Newman, Class. Quantum Grav. 13, 461 (1996).

[73] I.S. Gradshteyn and I.M. Ryzhik, Tables of Integrals, Series and Products (Academic Press, New York, 1965). 